\title{
Semi-Classical Wavefront Set and Fourier Integral Operators
}

\author{
Ivana Alexandrova \\ Department of Mathematics, University of Toronto, Toronto, Ontario, Canada M5S 3G3, \\ alexandr@math.toronto.edu
}

July 26, 2004

\begin{abstract}
Here we define and prove some properties of the semi-classical wavefront set. We also define and study semi-classical Fourier integral operators and prove a generalization of Egorov's Theorem to manifolds of different dimensions.
\end{abstract}

Keywords and phrases: Wavefront set, Fourier integral operators, Egorov Theorem, Semiclassical analysis.

\section{Introduction}

In this article we define and establish some of the properties of the semi-classical wavefront set and semi-classical Fourier integral operators. The paper is organized as follows. In Section 2 we review some of the theory of semi-classical pseudodifferential operators, which we will use here. In Section [3 we gather the existing definitions of semi-classical wavefront set and show that they are equivalent. We furthe explore the properties of the semi-classical wavefront set in Section 3.1. In Section 4 we define and prove a characterization of global semi-classical Fourier integral operators as well as a generalization of Egorov's Theorem to manifolds of unequal dimensions.

\section{Preliminaries}

In this section we recall some of the elements of semi-classical analysis which we will use here. First we define two classes of symbols

$$
S_{2 n}^{m}(1)=\left\{a \in C^{\infty}\left(\mathbb{R}^{2 n} \times\left(0, h_{0}\right]\right): \forall \alpha, \beta \in \mathbb{N}^{n}, \sup _{(x, \xi, h) \in \mathbb{R}^{2 n} \times\left(0, h_{0}\right]} h^{m}\left|\partial_{x}^{\alpha} \partial_{\xi}^{\beta} a(x, \xi ; h)\right| \leq C_{\alpha, \beta}\right\}
$$

and

$S^{m, k}\left(T^{*} \mathbb{R}^{n}\right)=\left\{a \in C^{\infty}\left(T^{*} \mathbb{R}^{n} \times\left(0, h_{0}\right]\right): \forall \alpha, \beta \in \mathbb{N}^{n},\left|\partial_{x}^{\alpha} \partial_{\xi}^{\beta} a(x, \xi ; h)\right| \leq C_{\alpha, \beta} h^{-m}\langle\xi\rangle^{k-|\beta|}\right\}$,

where $h_{0} \in(0,1]$ and $m, k \in \mathbb{R}$. For $a \in S_{2 n}(1)$ or $a \in S^{m, k}\left(T^{*} \mathbb{R}^{n}\right)$ we define the corresponding semi-classical pseudodifferential operator of class $\Psi_{h, t}^{m}\left(1, \mathbb{R}^{n}\right)$ or $\Psi_{h, t}^{m, k}\left(\mathbb{R}^{n}\right)$, respectively, by setting

$$
O p_{h}(a) u(x)=\frac{1}{(2 \pi h)^{n}} \iint e^{\frac{i\langle x-y, \xi\rangle}{h}} a(x, \xi ; h) u(y) d y d \xi, u \in \mathcal{S}\left(\mathbb{R}^{n}\right)
$$


for $t \in[0,1]$ and extending the definition to $\mathcal{S}^{\prime}\left(\mathbb{R}^{n}\right)$ by duality (see [2]). Below we shall work only with symbols which admit asymptotic expansions in $h$ and with pseudodifferential operators which are quantizations of such symbols. For $A \in \Psi_{h, t}^{k}\left(1, \mathbb{R}^{n}\right)$ or $A \in \Psi_{h, t}^{m, k}\left(\mathbb{R}^{n}\right)$, we shall use $\sigma_{0}(A)$ and $\sigma(A)$ to denote its principal symbol and its complete symbol, respectively.

For $a \in S_{n}^{m, k}\left(T^{*} \mathbb{R}^{n}\right)$ we define:

$$
\begin{aligned}
& \text { ess-supp }_{h} a \\
& =\left\{(x, \xi) \in T^{*} X \mid \exists \epsilon>0 \partial_{x}^{\alpha} \partial_{\xi}^{\beta} a\left(x^{\prime}, \xi^{\prime}\right)=\mathcal{O}_{C(B((x, \xi), \epsilon))}\left(h^{\infty}\right), \forall \alpha, \beta \in \mathbb{N}^{n}\right\}^{c} \\
& \cup\left(\left\{(x, \xi) \in T^{*} X \backslash\{0\} \mid \exists \epsilon>0 \partial_{x}^{\alpha} \partial_{\xi}^{\beta} a\left(x^{\prime}, \xi^{\prime}\right)=\mathcal{O}\left(h^{\infty}\langle\xi\rangle^{-\infty}\right),\right.\right. \\
& \left.\left.\quad \text { uniformly in }\left(x^{\prime}, \xi^{\prime}\right) \text { such that }\left\|x-x^{\prime}\right\|+\frac{1}{\left|\xi^{\prime}\right|}+\left|\frac{\xi}{|\xi|}-\frac{\xi^{\prime}}{\left|\xi^{\prime}\right|}\right|<\epsilon\right\} / \mathbb{R}_{+}\right)^{c} \\
& \subset T^{*} X \sqcup S^{*} X,
\end{aligned}
$$

where we define $S^{*} X=\left(T^{*} X \backslash\{0\}\right) / \mathbb{R}_{+}$. For $A \in \Psi_{h}^{m, k}\left(\mathbb{R}^{n}\right)$, we then define

$$
W F_{h}(A)=\operatorname{ess}^{-\operatorname{supp}_{h}} a, A=O p_{h}(a) .
$$

We also define the class of semi-classical distributions $\mathcal{D}_{h}^{\prime}\left(\mathbb{R}^{n}\right)$ with which we will work here

$$
\begin{aligned}
\mathcal{D}_{h}^{\prime}\left(\mathbb{R}^{n}\right)=\left\{u \in C_{h}^{\infty}\left((0,1] ; \mathcal{D}^{\prime}\left(\mathbb{R}^{n}\right)\right): \forall \chi \in C_{c}^{\infty}\left(\mathbb{R}^{n}\right) \exists N \in \mathbb{N} \text { and } C_{N}>0:\right. \\
\left.\left|\mathcal{F}_{h}(\chi u)(\xi, h)\right| \leq C_{N} h^{-N}\langle\xi\rangle^{N}\right\}
\end{aligned}
$$

where

$$
\mathcal{F}_{h}(u)(\xi, h)=\int_{\mathbb{R}^{n}} e^{-\frac{i}{h}\langle x, \xi\rangle} u(x, h) d x
$$

with the obvious extension of this definition to $\mathcal{E}_{h}^{\prime}\left(\mathbb{R}^{n}\right)$. We shall work with the $L^{2}$ - based semi-classical Sobolev spaces $H^{s}\left(\mathbb{R}^{n}\right), s \in \mathbb{R}$, which consist of the distributions $u \in \mathcal{D}_{h}^{\prime}\left(\mathbb{R}^{n}\right)$ such that $\|u\|_{H^{s}\left(\mathbb{R}^{n}\right)}^{2}=\frac{1}{(2 \pi h)^{n}} \int_{\mathbb{R}^{n}}\left(1+\|\xi\|^{2}\right)^{s}\left|\mathcal{F}_{h}(u)(\xi, h)\right|^{2} d \xi<\infty$.

We shall say that $u=v$ microlocally near an open set $U \subset T^{*} \mathbb{R}^{n}$, if $P(u-v)=\mathcal{O}\left(h^{\infty}\right)$ in $C_{c}^{\infty}\left(\mathbb{R}^{n}\right)$ for every $P \in \Psi_{h}^{0}\left(1, \mathbb{R}^{n}\right)$ such that

$$
W F_{h}(P) \subset \tilde{U}, \bar{U} \Subset \tilde{U} \Subset T^{*} \mathbb{R}^{n}, \tilde{U} \text { open. }
$$

We shall also say that $u$ satisfies a property $\mathcal{P}$ microlocally near an open set $U \subset T^{*} \mathbb{R}^{n}$ if there exists $v \in \mathcal{D}_{h}^{\prime}\left(\mathbb{R}^{n}\right)$ such that $u=v$ microlocally near $U$ and $v$ satisfies property $\mathcal{P}$.

For open sets $U, V \subset T^{*} \mathbb{R}^{n}$, the operators $T, T^{\prime} \in \Psi_{h}^{m}\left(\mathbb{R}^{n}\right)$ are said to be microlocally equivalent near $V \times U$ if for any $A, B \in \Psi_{h}^{0}\left(\mathbb{R}^{n}\right)$ such that

$$
\begin{gathered}
W F_{h}(A) \subset \tilde{V}, W F_{h}(B) \subset \tilde{U}, \bar{V} \Subset \tilde{V} \Subset T^{*} \mathbb{R}^{n}, \bar{U} \Subset \tilde{U} \Subset T^{*} \mathbb{R}^{n}, \tilde{U}, \tilde{V} \text { open } \\
A\left(T-T^{\prime}\right) B=\mathcal{O}\left(h^{\infty}\right): \mathcal{D}_{h}^{\prime}\left(\mathbb{R}^{n}\right) \rightarrow C^{\infty}\left(\mathbb{R}^{n}\right) .
\end{gathered}
$$

We shall also use the notation $T \equiv T^{\prime}$. 


\section{Semi-Classical Wavefront Set}

In this section we discuss the different notions of semi-classical wavefront set used in the literature and show that they are equivalent. We further establish some of their properties.

We further let $\hat{T}^{*} \mathbb{R}^{n}=T^{*} \mathbb{R}^{n} \sqcup S^{*} \mathbb{R}^{n}$, where we set $S^{*} \mathbb{R}^{n}=\left(T^{*} \mathbb{R}^{n} \backslash 0\right) / \mathbb{R}_{+}$with the $\mathbb{R}_{+}$ action given by mutiplication on the fibers: $(x, \xi) \mapsto(x, t \xi)$. As in [6], the points in $T^{*} \mathbb{R}^{n}$ will be called finite and the points in $S^{*} \mathbb{R}^{n}$ will be called infinite. We make the following definition as in [6]

Definition 1 Let $u \in \mathcal{D}_{h}^{\prime}\left(\mathbb{R}^{n}\right)$ and let $\left(x_{0}, \xi_{0}\right) \in \hat{T}^{*}\left(\mathbb{R}^{n}\right)$. We shall say that $\left(x_{0}, \xi_{0}\right)$ does not belong to $W F_{h}(u)$ if:

- If $\left(x_{0}, \xi_{0}\right)$ is finite: there exist $\chi \in C_{c}^{\infty}\left(\mathbb{R}^{n}\right)$ with $\chi\left(x_{0}\right) \neq 0$ and an open neighborhood $U$ of $\xi_{0}$, such that $\forall N \in \mathbb{N}, \forall \xi \in U,|\mathcal{F}(\chi u)(\xi, h)| \leq C_{N} h^{N}$. We shall denote the complement of the set of all such points by $W F_{h}^{f}(u)$.

- If $\left(x_{0}, \xi_{0}\right)$ is infinite: there exist $\chi \in C_{c}^{\infty}\left(\mathbb{R}^{n}\right)$ with $\chi\left(x_{0}\right) \neq 0$ and a conic neighborhood $U$ of $\xi_{0}$, such that $\forall N \in \mathbb{N}, \forall \xi \in U \cap\left\{|\xi| \geq \frac{1}{C}\right\}$,

$$
|\mathcal{F}(\chi u)(\xi, h)| \leq C_{N} h^{N}\langle\xi\rangle^{-N}
$$

We shall denote the complement of the set of all such points by $W F_{h}^{i}(u)$.

The definition of semi-classical wavefront set given in [7] is as follows

\section{Definition 2}

$$
W F_{h}(u)=\left\{(x, \xi): \exists A \in \Psi_{h}^{0,0}\left(\mathbb{R}^{n}\right) \sigma_{o}(A)(x, \xi) \neq 0, A u \in h^{\infty} C^{\infty}\left((0,1]_{h} ; C^{\infty}\left(\mathbb{R}^{n}\right)\right)\right\}^{c} .
$$

Lemma $1 u \in \mathcal{D}_{h}^{\prime}\left(\mathbb{R}^{n}\right)$ if and only if for every $\chi \in C_{c}^{\infty}\left(\mathbb{R}^{n}\right)$ there exist $m, k_{m}, C_{m} \in \mathbb{R}$ such that for every $\|\chi u\|_{H^{m}\left(\mathbb{R}^{n}\right)} \leq C_{m} h^{-k_{m}}$.

Proof: The first implication is clear. For the second implication, let $s \in \mathbb{R}$ be such that $m+s>\frac{n}{2}$ and consider

$$
\begin{aligned}
& C_{m}^{2} h^{-2 k_{m}} \geq \frac{1}{(2 \pi h)^{n}} \int\left(1+|\xi|^{2}\right)^{m}\left|\mathcal{F}_{h}(\chi u)(\xi, h)\right|^{2} d \xi \\
& =\frac{1}{(2 \pi h)^{n}} \int\left(1+|\xi|^{2}\right)^{m+s} \frac{\left|\mathcal{F}_{h}(\chi u)(\xi, h)\right|^{2}}{\left(1+|\xi|^{2}\right)^{s}} d \xi=\|\tilde{u}\|_{H^{m+s}\left(\mathbb{R}^{n}\right)}^{2},
\end{aligned}
$$

where $\tilde{u}=\mathcal{F}_{h}^{-1}\left(\frac{\mid \mathcal{F}_{h}(\chi u)(\cdot, h)}{\left(1+|\cdot|^{2}\right)^{\frac{s}{2}}}\right)$. Then we have that

$$
C_{m} h^{-k_{m}} \geq\|\tilde{u}\|_{H^{m+s}\left(\mathbb{R}^{n}\right)} \geq C\|\tilde{u}\|_{L^{\infty}\left(\mathbb{R}^{n}\right)} \geq C \frac{1}{|\operatorname{supp} \chi|}\|\hat{\tilde{u}}\|_{L^{\infty}\left(\mathbb{R}^{n}\right)}
$$

and therefore

$$
\left|\mathcal{F}_{h}(\chi u)(\xi, h)\right| \leq C h^{-k_{m}}\left(1+|\xi|^{2}\right)^{\frac{s}{2}}
$$


Lemma 2 Definitions (1) and (2) are equivalent.

Proof: Let $\left(x_{0}, \xi_{0}\right) \in T^{*} \mathbb{R}^{n} \backslash W F_{h}^{f}(u)$. Let $\varphi \in C_{c}^{\infty}\left(\mathbb{R}^{n}\right)$ satisfy $\varphi\left(x_{0}\right) \neq 0$ and let $\chi \in$ $C_{c}^{\infty}\left(\mathbb{R}^{n}\right)$ have support in a bounded open neighborhood $V$ of $\xi_{0}$ such that $\mathcal{F}_{h}(\varphi u)(\xi)=$ $\mathcal{O}\left(h^{\infty}\right)$ uniformly for $\xi \in V$. Consider

$$
A u(x)=\frac{1}{(2 \pi h)^{n}} \iint e^{\frac{i}{h}\langle x-y, \xi\rangle} \varphi(x) \varphi(y) \chi(\xi) u(y) d y d \xi .
$$

We clearly have that $A \in \Psi_{h}^{0,0}\left(\mathbb{R}^{n}\right)$ with $\sigma_{0}^{l}(A)\left(x_{0}, \xi_{0}\right) \neq 0$ and $A u=\mathcal{O}\left(h^{\infty}\right)$ in $C^{\infty}\left(\mathbb{R}^{n}\right)$, where $\sigma_{0}^{l}(A)$ is the principal symbol of the left-quantization of $A$.

Let, now, $\left(x_{0}, \xi_{0}\right) \in T^{*} \mathbb{R}^{n}$ be such that there exists $A \in \Psi_{h}^{0,0}\left(\mathbb{R}^{n}\right)$ elliptic at $\left(x_{0}, \xi_{0}\right)$ such that $A u=\mathcal{O}\left(h^{\infty}\right)$ in $C^{\infty}\left(\mathbb{R}^{n}\right)$. Let $\varphi, \chi \in C_{c}^{\infty}\left(\mathbb{R}^{n}\right)$ be such that $\varphi\left(x_{0}\right) \neq 0, \chi\left(\xi_{0}\right) \neq 0$, and $\chi(h D) \varphi=B A+R$, where $B \in \Psi_{h}^{0,0}\left(\mathbb{R}^{n}\right), R \in \Psi_{h}^{-\infty,-\infty}\left(\mathbb{R}^{n}\right)$. Then

$$
\begin{aligned}
\chi(h D) \varphi u(x) & =\frac{1}{(2 \pi h)^{n}} \iint e^{\frac{i}{h}\langle x-y, \xi\rangle} \chi(\xi) \varphi(y) u(y) d y d \xi \\
& =\frac{1}{(2 \pi h)^{n}} \int e^{\frac{i}{h} x \cdot \xi} \chi(\xi) \widehat{\varphi u}(\xi / h) d \xi=\mathcal{O}\left(h^{\infty}\right) \text { in } C^{\infty}\left(\mathbb{R}^{n}\right) .
\end{aligned}
$$

Therefore, $\chi(\xi) \widehat{\varphi u}(\xi / h)=\mathcal{O}\left(h^{\infty}\right)$ uniformly in $\xi$ and therefore $\widehat{\varphi u}(\xi / h)=\mathcal{O}\left(h^{\infty}\right)$ uniformly in $\xi$ in a bounded open set containing $\xi_{0}$, which implies that $\left(x_{0}, \xi_{0}\right) \notin W F_{h}^{f}(u)$.

The case of an infinite point is handled similarly. See also [3] for the proof in the classical setting, which applies directly to the infinite semi-classical wavefront set here.

\subsection{Properties of the Semi-classical Wavefront Set}

In this section, we prove the following properties of the semi-classical wavefront set

Lemma 3 Let $u \in \mathcal{D}_{h}^{\prime}\left(\mathbb{R}^{d_{1}}\right), v \in \mathcal{D}_{h}^{\prime}\left(\mathbb{R}^{d_{2}}\right), w \in \mathcal{D}_{h}^{\prime}\left(\mathbb{R}^{d_{3}}\right), V \in \mathcal{D}_{h}^{\prime}\left(\mathbb{R}^{d_{1}+d_{2}}\right), W \in \mathcal{D}_{h}^{\prime}\left(\mathbb{R}^{d_{2}+d_{3}}\right)$. Then

(a) $W F_{h}^{f}(A u) \subset W F_{h}^{f}(A) \cap W F_{h}^{f}(u)$ and $W F_{h}^{i}(A u) \subset W F_{h}^{i}(A) \cap W F_{h}^{i}(u)$, for $A \in$ $\Psi_{h}^{m, k}\left(\mathbb{R}^{d_{1}}\right)$.

(b) $u \otimes v \in \mathcal{D}_{h}^{\prime}\left(\mathbb{R}^{d_{1}+d_{2}}\right), W F_{h}^{f}(u \otimes v) \subset W F_{h}^{f}(u) \times W F_{h}^{f}(v)$ and

$$
\begin{gathered}
W F_{h}^{i}(u \otimes v) \subset\left(W F_{h}^{i}(u) \times W F_{h}^{i}(v)\right) \cup\left((\operatorname{supp} u \times\{0\}) \times W F_{h}^{i}(v)\right) \\
\cup\left(W F_{h}^{i}(u) \times(\operatorname{supp} v \times\{0\})\right) .
\end{gathered}
$$

(c) if $V$ is proper, $W F_{h}^{i}(v) \cap\left(W F_{h}^{i}\right)_{\mathbb{R}^{d_{2}}}^{\prime}(V)=\emptyset$, and $W F_{h}^{f}(v)$ is compact, then $V v \in \mathcal{D}_{h}^{\prime}\left(\mathbb{R}^{d_{1}}\right)$ and

$$
W F_{h}^{i}(V v) \subset\left(W F_{h}^{i}\right)^{\prime}(V)\left(W F_{h}^{i}(v)\right) \cup\left(W F_{h}^{i}\right)_{\mathbb{R}^{d_{1}}}^{\prime}(V)
$$


and

$$
W F_{h}^{f}(V v) \subset\left(W F_{h}^{f}\right)^{\prime}(V)\left(W F_{h}^{f}(v)\right)
$$

where

$$
\left(W F_{h}^{i}\right)_{\mathbb{R}^{d_{2}}}^{\prime}(V)=\left\{(y, \eta) \in T^{*} \mathbb{R}^{d_{2}} \backslash\{0\}: \exists x \in \mathbb{R}^{d_{1}},(x, 0 ; y, \eta) \in\left(W F_{h}^{i}\right)^{\prime}(V)\right\}
$$

and $V v$ is defined as in Theorem 7.8, [3]. The same conclusion holds if $V$ is not necessarily proper but $v \in \mathcal{E}_{h}^{\prime}\left(\mathbb{R}^{d_{2}}\right)$ and all the other assumptions are satisfied.

(d) if at least one of $V$ and $W$ are properly supported,

$$
\begin{gathered}
\left(W F_{h}^{i}\right)_{\mathbb{R}^{d_{2}}}^{\prime}(V) \cap\left(W F_{h}^{i}\right)_{\mathbb{R}^{d_{2}}}^{\prime}(W)=\emptyset \\
\left\{p \in \mathbb{R}^{d_{2}}: \exists(q, r) \in \mathbb{R}^{d_{1}} \times \mathbb{R}^{d_{3}},(q, p) \in W F_{h}^{f}(V),(p, r) \in W F_{h}^{f}(W)\right\}
\end{gathered}
$$

is compact, then $V \circ W \in \mathcal{D}_{h}^{\prime}\left(\mathbb{R}^{d_{1}+d_{3}}\right)$,

$$
\left(W F_{h}^{i}\right)^{\prime}(V \circ W) \subset\left(W F_{h}^{i}\right)^{\prime}(V) \circ\left(W F_{h}^{i}\right)^{\prime}(W),
$$

and

$$
\left(W F_{h}^{f}\right)^{\prime}(V \circ W) \subset\left(W F_{h}^{f}\right)^{\prime}(V) \circ\left(W F_{h}^{f}\right)^{\prime}(W),
$$

where $V \circ W$ is defined as in Theorem 7.10, [3].

Remark. Part (c) of this lemma is proved in [1, Proposition A.I.13] without the assumption on $W F_{h}(v)$. In our proof, however, we also show that all estimates can be made uniformly in a neighborhood of $W F_{h}(v)$.

Proof: In this proof we shall use $\langle\cdot, \cdot\rangle$ to denote the distribution pairing. We begin by proving (a). Let $\left(x_{0}, \xi_{0}\right) \notin W F_{h}(A)$ and assume that $\left(x_{0}, \xi_{0}\right)$ is a finite point. Let $B \in$ $\Psi_{h}^{0,0}\left(\mathbb{R}^{d_{1}}\right)$ satisfy $\sigma(B)\left(x_{0}, \xi_{0}\right) \neq 0, \sigma(B) \in C_{c}^{\infty}\left(\mathbb{R}^{d_{1}}\right)$, and $W F_{h}(B) \cap W F_{h}(A)=\emptyset$. Then $B A \in \Psi_{h}^{-\infty,-\infty}\left(\mathbb{R}^{d_{1}}\right)$ and therefore $B A u=\mathcal{O}\left(h^{\infty}\right)$ in $C^{\infty}\left(\mathbb{R}^{d_{1}}\right)$. If $\left(x_{0}, \xi_{0}\right)$ is an infinite point, we can again find $B \in \Psi_{h}^{0,0}\left(\mathbb{R}^{d_{1}}\right)$ with $W F_{h}(B)$ consisting only of infinite points such that $W F_{h}(B) \cap W F_{h}(A)=\emptyset$ and we then have that $B A u=\mathcal{O}\left(h^{\infty}\right)$ in $C^{\infty}\left(\mathbb{R}^{d_{1}}\right)$.

Let, now, $\left(x_{0}, \xi_{0}\right) \notin W F_{h}^{f}(u)$. Let $c \in C_{c}^{\infty}\left(\mathbb{R}^{d_{1}}\right)$ satisfy $c\left(x_{0}, \xi_{0}\right) \neq 0$ and let $d \in S_{2 d_{1}}(1)$ be such that $d \#_{h} c=1$ in a neighborhood $W \subset\left(W F_{h}^{f}(u)\right)^{c}$ of $\left(x_{0}, \xi_{0}\right)$. Further, let $\chi \in$ $C_{c}^{\infty}\left(T^{*} \mathbb{R}^{d_{1}}\right)$ have support in an open set $V \Subset W$ and be equal to 1 on an open subset $U \Subset V$. Then the operator $T=O p_{h}\left(\chi d \#_{h} c\right)$ has symbol $\sigma(T) \equiv 1 \bmod h^{\infty}$ in $S_{2 d_{1}}(1)$ in $U$ and supported in $W$ and therefore $T u=\mathcal{O}\left(h^{\infty}\right)$ in $C^{\infty}\left(\mathbb{R}^{d_{1}}\right)$. Let $B$ be elliptic at $\left(x_{0}, \xi_{0}\right)$ with $W F_{h}(B) \subset U$. Then we have that $B A \equiv B A T \bmod \Psi_{h}^{-\infty,-\infty}\left(\mathbb{R}^{d_{1}}\right)$ and hence $B A u \equiv B A T u=\mathcal{O}\left(h^{\infty}\right)$ in $C^{\infty}\left(\mathbb{R}^{d_{1}}\right)$. Therefore, $\left(x_{0}, \xi_{0}\right) \notin W F_{h}(A u)$. The proof is similar in the case of an infinite point $\left(x_{0}, \xi_{0}\right)$. 
We now turn to proving (b). It is trivial to check that $u \otimes v \in \mathcal{D}_{h}^{\prime}\left(\mathbb{R}^{d_{1}+d_{2}}\right)$. Let $\left(x_{0}, \xi_{0} ; y_{0}, \eta_{0}\right) \notin W F_{h}^{f}(u) \times W F_{h}^{f}(v)$ and let $O_{1}, O_{1}^{\prime} \subset \mathbb{R}^{d_{1}}$ and $O_{2}, O_{2}^{\prime} \subset \mathbb{R}^{d_{3}}$ be open neighbordhoods of $x_{0}, \xi_{0}, y_{0}$, and $\eta_{0}$, respectively, such that $O_{1} \times O_{1}^{\prime} \times O_{2} \times O_{2}^{\prime} \subset\left(W F_{h}^{f}(u) \times W F_{h}^{f}(v)\right)^{c}$. Without loss of generality, we can assume that $\left(x_{0}, \xi_{0}\right) \notin W F_{h}^{f}(u)$. Then there exists $\chi_{1} \in C_{c}^{\infty}\left(\mathbb{R}^{d_{1}}\right)$ with $\chi_{1}\left(x_{0}\right) \neq 0$ and a bounded open set $O_{1}^{\prime} \subset \mathbb{R}^{d_{1}}$ with $\xi_{0} \in O_{1}^{\prime}$ such that $\left|\mathcal{F}_{h}\left(\chi_{1} u\right)(\xi, h)\right|=\mathcal{O}\left(h^{\infty}\right)$ uniformly for $\xi \in O_{1}^{\prime}$. Let, now, $\chi_{2} \in C_{c}^{\infty}\left(\mathbb{R}^{d_{2}}\right)$ have support near $y_{0}$. Then $\left|\mathcal{F}_{h}(v)(\eta, h)\right| \leq C h^{-M}\langle\eta\rangle^{M}$ for some $C>0, M>0$, and therefore $\left|\mathcal{F}_{h}\left(\chi_{1} u \otimes \chi_{2} v\right)(\xi, \eta, h)\right|=\mathcal{O}\left(h^{\infty}\right)$ uniformly in $(\xi, \eta) \in O_{1}^{\prime} \times O_{2}^{\prime}$ for any open bounded $O_{2}^{\prime} \subset \mathbb{R}^{d_{2}}$ with $\eta \in O_{2}^{\prime}$. Therefore $\left(x_{0}, \xi_{0} ; y_{0}, \eta_{0}\right) \notin W F_{h}^{f}(u \otimes v)$.

The proof of the second assertion in (b) is as in the $C^{\infty}$ case. See Theorem 8.2.9, 4].

To establish (c) and (d), we first prove the following

Lemma 4 Let $u_{1} \in \mathcal{D}_{h}^{\prime}\left(\mathbb{R}^{n}\right)$ and $u_{2} \in \mathcal{E}_{h}^{\prime}\left(\mathbb{R}^{n}\right)$ satisfy $W F_{h}\left(u_{1}\right) \cap W F_{h}^{\prime}\left(u_{2}\right)=\emptyset$.

Then $\int u_{1} u_{2}=\mathcal{O}\left(h^{\infty}\right)$, where the integral is defined as in Proposition 7.6, [3].

Proof: For $u \in \mathcal{D}_{h}^{\prime}\left(\mathbb{R}^{n}\right)$ let

$$
\begin{aligned}
& \Sigma_{h}=\left\{\xi \in \mathbb{R}^{n}: \exists x \in \mathbb{R}^{n},(x, \xi) \in W F_{h}(u)\right\} \\
& \Sigma_{h}^{i}=\left\{\xi \in \mathbb{R}^{n}: \exists x \in \mathbb{R}^{n},(x, \xi) \in W F_{h}^{i}(u)\right\} \\
& \Sigma_{h}^{f}=\left\{\xi \in \mathbb{R}^{n}: \exists x \in \mathbb{R}^{n},(x, \xi) \in W F_{h}^{f}(u)\right\} \\
& \Sigma_{h}^{x}=\left\{\xi \in \mathbb{R}^{n}:(x, \xi) \in W F_{h}(u)\right\}
\end{aligned}
$$

We have that $\Sigma_{h}^{x}(u)=\lim _{\operatorname{supp} \phi \rightarrow\{x\}} \Sigma_{h}(\phi u)$. The proof is the same as in the classical case $(h=1)$ (see 4], Section 8.1). For every $x_{0} \in \mathbb{R}^{n}$ we can then find $\varphi \in C_{c}^{\infty}\left(\mathbb{R}^{n}\right)$ such that $\varphi\left(x_{0}\right) \neq 0$ and $\Sigma_{h}\left(\varphi u_{1}\right) \cap\left(-\Sigma_{h}\left(\varphi u_{2}\right)\right)=\emptyset$. By Proposition 7.6, [3], we have that

$$
\int \varphi u_{1} \varphi u_{2}=\frac{1}{(2 \pi h)^{n}} \int \widehat{\varphi u}_{1}\left(\frac{\xi}{h}\right) \widehat{\varphi u}_{2}\left(-\frac{\xi}{h}\right) d \xi .
$$

Now, since $\Sigma_{h}^{i}\left(\varphi u_{1}\right) \cap \Sigma_{h}^{i}\left(\varphi u_{2}\right)=\emptyset$, for every $\xi_{0} \in \Sigma_{h}^{i}\left(\varphi u_{1}\right)$ we can find an open conic neighborhood $U_{\xi_{0}}$ of $\xi_{0}$ such that $\widehat{\varphi u_{2}}\left(-\frac{\xi}{h}\right)=\mathcal{O}\left(h^{\infty}\langle\xi\rangle^{-\infty}\right)$ uniformly in $U_{\xi_{0}} \cap\left\{\xi:\|\xi\| \geq \frac{1}{C}\right\}$, for some $C>0$. Since $u_{1} \in \mathcal{D}_{h}^{\prime}\left(\mathbb{R}^{n}\right)$, it follows that there exist $N \in \mathbb{N}$ and $C^{\prime}>0$ such that $\left|\mathcal{F}_{h}\left(\varphi u_{1}\right)(\xi, h)\right| \leq C^{\prime} h^{-N}\langle\xi\rangle^{N}$ and therefore $\widehat{\varphi u_{1}}\left(\frac{\xi}{h}\right) \widehat{\varphi u_{2}}\left(-\frac{\xi}{h}\right)=\mathcal{O}\left(h^{\infty}\langle\xi\rangle^{-\infty}\right)$ uniformly in $U_{\xi_{0}} \cap\left\{\xi:\|\xi\| \geq \frac{1}{C}\right\}$. The compactness of $\mathbb{S}^{n-1}$ implies that we can find finitely many such neighborhoods $\left(U_{l}^{1}\right)_{l=1}^{L_{1}}$ and $\left(U_{l}^{2}\right)_{l=1}^{L_{2}}$ and a constant $C_{1}>0$ satisfying $\Sigma_{h}^{i}\left(\varphi u_{1}\right) \subset \cup_{l=1}^{L_{2}} U_{l}^{2}$ and $\Sigma_{h}^{i}\left(\varphi u_{2}\right) \subset \cup_{l=1}^{L_{1}} U_{l}^{1}$ and such that $\widehat{\varphi u_{1}}\left(\frac{\xi}{h}\right) \widehat{\varphi u_{2}}\left(-\frac{\xi}{h}\right)=\mathcal{O}\left(h^{\infty}\langle\xi\rangle^{-\infty}\right)$ uniformly in $U_{l}^{j} \cap$ $\left\{\xi:\|\xi\| \geq \frac{1}{C}\right\}, l=1, \ldots, L_{j}, j=1,2$. We can further arrange to have $\left(\cup_{l=1}^{L_{1}} U_{l}^{1}\right) \cap\left(\cup_{l=1}^{L_{2}} U_{l}^{2}\right)=$ $\emptyset$. Lastly, we choose finitely many sets $\left(U_{l}\right)_{l=1}^{L_{3}}$ such that $\mathbb{S}^{n-1} \backslash\left(\cup_{k=1}^{2} \cup_{l}^{L_{k}} U_{l}^{k}\right) \subset \cup_{l=1}^{L_{3}} U_{l}$ and a constant $C_{2}>0$ such that $\widehat{\varphi u_{j}}\left(\frac{\xi}{h}\right)=\mathcal{O}\left(h^{\infty}\langle\xi\rangle^{-\infty}\right)$ uniformly in $\xi \in\left\{\xi:\|\xi\|>\frac{1}{C_{2}}\right\} \cap U_{l}$, $j=1,2, l=1, \ldots, L_{3}$. With $C=\min \left\{C_{1}, C_{2}\right\}$, we then have

$$
\int_{\left\{\xi:\|\xi\|>\frac{1}{C}\right\}} \widehat{\varphi u}_{1}\left(\frac{\xi}{h}\right) \widehat{\varphi u}_{2}\left(-\frac{\xi}{h}\right) d \xi=\mathcal{O}\left(h^{\infty}\right) .
$$


The same argument applied now to $\Sigma_{h}^{f}\left(\varphi u_{j}\right) \cap\left\{\xi \in \mathbb{R}^{n}:\|\xi\| \leq \frac{1}{C}\right\}, j=1,2$, gives that

$$
\int_{\left\{\xi:\|\xi\| \leq \frac{1}{C}\right\}} \widehat{\varphi u}_{1}\left(\frac{\xi}{h}\right) \widehat{\varphi u}_{2}\left(-\frac{\xi}{h}\right) d \xi=\mathcal{O}\left(h^{\infty}\right)
$$

and therefore

$$
\int \varphi u_{1} \varphi u_{2}=\mathcal{O}\left(h^{\infty}\right)
$$

Choosing a locally finite partition of unity $\sum_{j=1}^{\infty} \varphi_{j}^{2}=1$ with each function $\varphi_{j}$ chosen as $\varphi$ above, we have that $\left\langle u_{1}, u_{2}\right\rangle=\sum_{j=1}^{\infty}\left\langle\varphi_{j} u_{1}, \varphi_{2} u_{2}\right\rangle=\mathcal{O}\left(h^{\infty}\right)$.

We, now, turn to proving (c). The fact that $V v \in \mathcal{D}_{h}^{\prime}\left(\mathbb{R}^{d_{1}}\right)$ is proved in [1] Proposition A.I.13]. We shall now prove that $\left(W F_{h}^{f}\right)^{\prime}(V)\left(W F_{h}^{f}(v)\right)$ is a closed set. Let $\left(\left(x_{n}, \xi_{n}\right)\right)_{n \in \mathbb{N}} \subset$ $\left(W F_{h}^{f}\right)^{\prime}(V)\left(W F_{h}^{f}(v)\right)$ converge to $\left(x_{0}, \xi_{0}\right)$. For every $n \in \mathbb{N}$ let $\left(y_{n}, \eta_{n}\right) \in W F_{h}^{f}(v)$ be such that $\left(x_{n}, \xi_{n} ; y_{n}, \eta_{n}\right) \in\left(W F_{h}^{f}\right)^{\prime}(V)$. Since $W F_{h}^{f}(v)$ is compact, after passing to a subsequence, we can assume that $\left(y_{n}, \eta_{n}\right) \rightarrow\left(y_{0}, \eta_{0}\right) \in W F_{h}^{f}(v)$. Therefore $\left(x_{n}, \xi_{n} ; y_{n}, \eta_{n}\right) \rightarrow\left(x_{0}, \xi_{0} ; y_{0}, \eta_{0}\right)$ and since $\left(W F_{h}^{f}\right)^{\prime}(V)$ is closed, it follows that $\left(x_{0}, \xi_{0} ; y_{0}, \eta_{0}\right) \in\left(W F_{h}^{f}\right)^{\prime}(V)$. This implies that $\left(x_{0}, \xi_{0}\right) \in\left(W F_{h}^{f}\right)^{\prime}(V)\left(W F_{h}^{f}(v)\right)$ and therefore $\left(W F_{h}^{f}\right)^{\prime}(V)\left(W F_{h}^{f}(v)\right)$ is closed.

Let, now, $\left(x_{0}, \xi_{0}\right) \in\left(\left(W F_{h}^{f}\right)^{\prime}(V)\left(W F_{h}^{f}(v)\right)\right)^{c}$ and let $O, O^{\prime} \subset \mathbb{R}^{d_{1}}$ be open neighborhoods of $x_{0}$ and $\xi_{0}$, respectively, such that

$$
O \times O^{\prime} \subset\left(\left(W F_{h}^{f}\right)^{\prime}(V)\left(W F_{h}^{f}(v)\right)\right)^{c}
$$

and $O^{\prime}$ is bounded. Let $\chi \in C_{c}^{\infty}\left(\mathbb{R}^{d_{1}}\right)$ have support in $O$ and let $\xi \in O^{\prime}$. By the proof of Lemma 4, we have that

$$
\left\langle V(\cdot, \cdot \cdot), \chi(\cdot) e^{-\frac{i}{h}\langle\cdot, \xi\rangle} \otimes v(\cdot \cdot)\right\rangle=\frac{1}{(2 \pi h)^{d_{1}+d_{2}}}\left\langle\hat{V}, \chi\left(\widehat{\left.\cdot) e^{-\frac{i}{h} l} \cdot, \xi\right\rangle} \otimes \hat{v}\right\rangle=\mathcal{O}\left(h^{\infty}\right)\right.
$$

uniformly in $\xi \in O^{\prime}$.

The proof in the case of the infinite wave front set is the same.

Lastly, to prove (d), we first observe that the fact that $V \circ W \in \mathcal{D}_{h}^{\prime}\left(\mathbb{R}^{d_{1}+d_{3}}\right)$ follows as in the proof of [1, Proposition A.I.13]. To establish (c), now, we begin by proving that $\left(W F_{h}^{f}\right)^{\prime}(V) \circ\left(W F_{h}^{f}\right)^{\prime}(W)$ is closed. For that, let

$$
\left(\left(x_{n}, \xi_{n} ; y_{n}, \eta_{n}\right)\right)_{n \in \mathbb{N}} \subset\left(W F_{h}^{f}\right)^{\prime}(V) \circ\left(W F_{h}^{f}\right)^{\prime}(W
$$

converge to $\left(x_{0}, \xi_{0} ; y_{0}, \eta_{0}\right)$. Let $\left(\left(z_{n}, \zeta_{n}\right)\right)_{n \in \mathbb{N}} \subset \mathbb{R}^{m}$ be such that $\left(x_{n}, \xi_{n} ; z_{n}, \zeta_{n}\right) \in\left(W F_{h}^{f}\right)^{\prime}(V)$, $\left(z_{n}, \zeta_{n} ; y_{n}, \eta_{n}\right) \in\left(W F_{h}^{f}\right)^{\prime}(W), n \in \mathbb{N}$. By the assumption, we can assume that, after passing to a subsequence, $\left(z_{n}, \zeta_{n}\right) \rightarrow\left(z_{0}, \zeta_{0}\right)$. Since then $\left(x_{n}, \xi_{n} ; z_{n}, \zeta_{n}\right) \rightarrow\left(x_{0}, \xi_{0} ; z_{0}, \zeta_{0}\right)$ and 
$\left(W F_{h}^{f}\right)^{\prime}(V)$ is closed, it follows that $\left(x_{0}, \xi_{0} ; z_{0}, \zeta_{0}\right) \in\left(W F_{h}^{f}\right)^{\prime}(V)$. Similarly, $\left(y_{0}, \eta_{0} ; z_{0}, \zeta_{0}\right) \in$ $\left(W F_{h}^{f}\right)^{\prime}(W)$, and therefore

$$
\left(x_{0}, \xi_{0} ; y_{0}, \eta_{0}\right) \in\left(W F_{h}^{f}\right)^{\prime}(V) \circ\left(W F_{h}^{f}\right)^{\prime}(W) .
$$

Let, now, $(x, \xi ; y, \eta) \in\left(\left(W F_{h}^{f}\right)^{\prime}(V) \circ\left(W F_{h}^{f}\right)^{\prime}(W)\right)^{c}$. Let $O_{1}, O_{1}^{\prime} \subset \mathbb{R}^{d_{1}}, O_{2}, O_{2}^{\prime} \subset \mathbb{R}^{d_{3}}$ be open neighborhoods of $x, \xi, y$, and $\eta$, respectively, such that $O_{1} \times O_{1}^{\prime} \times O_{2} \times O_{2}^{\prime} \subset$ $\left(W F_{h}^{\prime}(V) \circ W F_{h}^{\prime}(W)\right)^{c}$ and $O_{1}^{\prime}$ and $O_{2}^{\prime}$ are bounded. Let $\varphi \in C_{c}^{\infty}\left(\mathbb{R}^{d_{1}}\right), \psi \in C_{c}^{\infty}\left(\mathbb{R}^{d_{3}}\right)$ have supports inside $O_{1}$ and $O_{2}$, respectively. Then, by the proof of Lemma 4 , we have that

$$
\left\langle V(\cdot, \cdot \cdot) \otimes \psi(\cdots) e^{-\frac{i}{h}\langle\cdots, \eta\rangle}, \varphi(\cdot) e^{-\frac{i}{h}\langle\cdot \xi\rangle} \otimes W(\cdot \cdot, \cdot \cdot)\right\rangle=\mathcal{O}\left(h^{\infty}\right)
$$

uniformly in $(\xi, \eta) \in O_{1}^{\prime} \times O_{2}^{\prime}$. Therefore $(x, \xi ; y, \eta) \notin W F_{h}^{f}(V \circ W)$.

The proof in the infinite case is the same as in the $C^{\infty}$ case, see Theorem 7.10, [3].

\section{Global Semi-Classical Fourier Integral Operators}

Here we prove a characterization of global semi-classical Fourier Integral Operators, which is the semi-classical analog of Melrose's characterization of Lagrangian distributions in 4 , Definition 25.1.1].

\subsection{Parametrizing Lagrangian Submanifolds}

We first review some facts from symplectic geometry relating non-degenerate phase functions and Lagrangian submanifolds.

Let $V \subset \mathbb{R}^{n} \times \mathbb{R}^{m}, m \in \mathbb{N}_{0}$, be an open set and let $\varphi=\varphi(x, \theta) \in C_{b}^{\infty}(V ; \mathbb{R})$. For $m>0$, let $\varphi$ also be a phase function in the sense of [5], Section 2.4. If $a \in S_{n+m}$ (1), we define the oscillatory integral $I(a, \varphi)=\int_{\mathbb{R}^{m}} e^{\frac{i}{h} \varphi(\cdot, \theta)} a(\cdot, \theta) d \theta$ as in [5], Section 2.4 if $m>0$ and set $I(a, \varphi)=e^{\frac{i}{h} \varphi} a$ if $m=0$.

We further let

$$
C_{\varphi}=\left\{(x, \theta) \in V: \varphi_{\theta}^{\prime}(x, \theta)=0\right\}
$$

and

$$
\Lambda_{\varphi}=\left\{\left(x, \varphi_{x}^{\prime}(x, \theta)\right):(x, \theta) \in C_{\varphi}\right\}
$$

and recall that a phase function $\varphi$ is non-degenerate if

$$
\varphi_{\theta}^{\prime}(x, \theta)=0 \text { implies that }\left(\varphi_{\theta x}^{\prime \prime} \varphi_{\theta \theta}^{\prime \prime}\right) \text { has maximum rank at }(x, \theta) .
$$

If $m=0$, it is a standard fact from symplectic geometry that $\Lambda_{\varphi}$ is a Lagrangian submanifold of $T^{*}\left(\mathbb{R}^{n}\right)$. If $m>0$, (3) implies that $C_{\varphi}$ is a smooth $n$-dimensional manifold. Let $j_{\varphi}: C_{\varphi} \ni$ 
$(x, \theta) \mapsto\left(x, \varphi_{x}^{\prime}(x, \theta)\right) \in \Lambda_{\varphi}$. Then, after shrinking $V$ around any fixed point $\left(x^{\prime}, \theta^{\prime}\right) \in C_{\varphi}$, we can assume that $\Lambda_{\varphi}$ is a Lagrangian submanifold of $T^{*} \mathbb{R}^{n}$ and $j_{\varphi}$ is a diffeomorphism. For a proof, we refer the reader to [3], Lemmas 11.2 and 11.3.

If $\Lambda \subset T^{*} \mathbb{R}^{n}$ is a Lagrangian submanifold such that the map $\pi_{\xi}: \Lambda \cap U \ni(x, \xi) \mapsto \xi \in \mathbb{R}^{n}$ is a local diffeomorphism, then there exist an open set $W \subset \mathbb{R}^{n} \backslash\{0\}$ and a function $H \in$ $C_{b}^{\infty}(W ; \mathbb{R})$ satisfying

$$
\Lambda \cap U=\left\{\left(H^{\prime}(\xi), \xi\right): \xi \in W\right\} .
$$

For a proof, see [3], Section 9.

If $\Lambda \subset T^{*} \mathbb{R}^{n}$ is any Lagrangian submanifold and $\gamma \in \Lambda$, then there exists an open set $U \subset T^{*} \mathbb{R}^{n}$ and a non-degenerate phase function $\varphi \in C^{\infty}(V), V \subset \mathbb{R}^{n+m}$ open, $m \in \mathbb{N}_{0}$ such that

$$
\Lambda \cap U=\Lambda_{\varphi}
$$

We include the proof of this well-known result here for completeness and to introduce some notation. Let $\mu=T_{\gamma} \Lambda$ be identified in a natural way with a subspace of $T^{*} \mathbb{R}^{n}$. By Lemma $9.5,3$. we have that after a linear change of coordinates we may assume that

$$
\mu=\left\{\left(0, x^{\prime \prime} ; \xi^{\prime}, B x^{\prime \prime}\right)\right\}
$$

for a splitting of the coordinates $x=\left(x^{\prime}, x^{\prime \prime}\right)$ and $\xi=\left(\xi^{\prime}, \xi^{\prime \prime}\right)$, where $x^{\prime}=\left(x_{1}, \ldots, x_{k}\right), k=$ $0, \ldots, n$, and $B$ is a real symmetric matrix. This implies that the differential of the projection $\pi: \Lambda \rightarrow\left(x^{\prime \prime}, \xi^{\prime}\right)$ is bijective at $\gamma$ and therefore this map is a local diffeomorphism from a neighborhood of $\gamma$ to the $\left(x^{\prime \prime}, \xi^{\prime}\right)$-space. Therefore there exists a function $S \in C^{\infty}\left(\mathbb{R}^{n} ; \mathbb{R}\right)$

and an open neighborhood $U \subset T^{*} \mathbb{R}^{n}$ of $\gamma$ such that $\Lambda \cap U=\left\{\left(\frac{\partial S}{\partial \xi^{\prime}}, x^{\prime \prime} ; \xi^{\prime},-\frac{\partial S}{\partial x^{\prime \prime}}\right)\right\} \cap U$. From this it easily follows that $\varphi\left(x, \xi^{\prime}\right)=\left\langle x^{\prime}, \xi^{\prime}\right\rangle-S\left(x^{\prime \prime}, \xi^{\prime}\right)$ is a non-degenerate phase function such that $\Lambda \cap U=\Lambda_{\varphi} \cap U$.

\subsection{Semi-Classical Fourier Intergal Operators}

We are now ready to make the following definition

Definition 3 Let $M$ be a smooth $k$-dimensional manifold and let $\Lambda \subset T^{*} M$ be a smooth closed Lagrangian submanifold with respect to the canonical symplectic structure on $T^{*} M$. Let $r \in \mathbb{R}$. Then the space $I_{h}^{r}(M, \Lambda)$ of semi-classical Fourier integral distributions of order $r$ associated to $\Lambda$ is defined as the set of all $u \in \mathcal{D}_{h}^{\prime}(M)$ such that

$$
\left(\prod_{j=0}^{N} A_{j}\right)(u)=\mathcal{O}_{L^{2}(M)}\left(h^{N-r-\frac{k}{4}}\right), h \rightarrow 0,
$$

for all $N \in \mathbb{N}_{0}$ and for all $A_{j} \in \Psi_{h}^{0}(1, X), j=0, \ldots, N-1$, with compactly supported symbols and principal symbols vanishing on $\Lambda$, and any $A_{N} \in \Psi_{h}^{0}(1, X)$ with a compactly supported symbol. 
A continuous linear operator $C_{c}^{\infty}\left(M_{1}\right) \rightarrow \mathcal{D}_{h}^{\prime}\left(M_{2}\right)$, where $M_{1}, M_{2}$ are smooth manifolds, whose Schwartz kernel is an element of $I_{h}^{r}\left(M_{1} \times M_{2}, \Lambda\right)$ for some Lagrangian submanifold $\Lambda \subset T^{*} M_{1} \times T^{*} M_{2}$ and some $r \in \mathbb{R}$ will be called a global semi-classical Fourier integral operator of order $r$ associated to $\Lambda$. We denote the space of these operators by $\mathcal{I}_{h}^{r}\left(M_{1} \times M_{2}, \Lambda\right)$.

Remark: The exotic looking numerology for the order needs to be explained. We follow the same convention as that in classical case and require that pseudodifferential operators with compactly supported symbols in $S^{0}(1)$ have kernels in $I_{h}^{0}\left(\mathbb{R}^{2 n}, N^{*} \Delta\right)$, where $\Delta$ is the diagonal in $\mathbb{R}^{2 n}$. Explicitly, suppose that

$$
K(x, y)=\frac{1}{(2 \pi h)^{n}} \int e^{\frac{i}{h}\langle x-y, \xi\rangle} c(x, \xi) d \xi, \quad c \in S^{0}(1) \cap C_{\mathrm{c}}^{\infty}\left(\mathbb{R}^{2 n}\right) .
$$

Then

$$
\|K\|_{L^{2}\left(\mathbb{R}^{2 n}\right)}=\frac{1}{(2 \pi h)^{\frac{n}{2}}}\left\|\mathcal{F}_{h}^{y} K\right\|_{L^{2}\left(\mathbb{R}^{2 n}\right)}=\frac{1}{(2 \pi h)^{\frac{n}{2}}}\|c\|_{L^{2}\left(\mathbb{R}^{2 n}\right)},
$$

where $\mathcal{F}_{h}^{y}$ is the semi-classical Fourier transform in the $y$ variable, which is consistent with (17) with $N=0$ and the order $r=0$ ( $k=2 n$ here).

We, now, have the following semi-classical analog of [4, Lemma 25.1.2], vol. IV.

Lemma 5 If $u \in I_{h}^{r}(M, \Lambda)$, then $A u \in I_{h}^{r}(M, \Lambda)$ for every $A \in \Psi_{h}^{0}(1, M)$ or $A \in \Psi_{h}^{0, k}\left(T^{*} M\right)$, $k \in \mathbb{R}$ with compactly supported symbol.

If $u \in \mathcal{D}_{h}^{\prime}(M)$ is such that for every $\left(x_{0}, \xi_{0}\right) \in \Lambda$ there exists $A \in \Psi_{h}^{0}(1, M)$ elliptic at $\left(x_{0}, \xi_{0}\right)$ with compactly supported symbol and $A u \in I_{h}^{r}(M, \Lambda)$, then $u \in I_{h}^{r}(M, \Lambda)$. The same conclusion holds if $A \in \Psi_{h}^{0, k}\left(T^{*} M\right), k \in \mathbb{R}$.

Proof: To prove the first statement, let $u \in I_{h}^{r}(M, \Lambda)$, let $A \in \Psi_{h}^{0}(1, M)$ have a compactly supported symbol, and let $A_{j} \in \Psi_{h}^{0}(1, M), j=1, \ldots, N, N \in \mathbb{N}$, also have compactly supported symbols and principal symbols vanishing on $\Lambda$. Then

$$
\left(\prod_{j=1}^{N} A_{j}\right)(A u)=\left(\prod_{j=1}^{N-1} A_{j}\right)\left[A_{N}, A\right] u+\left(\prod_{j=1}^{N-1} A_{j}\right) A A_{N} u .
$$

Here $\left[A_{N}, A\right] \in \Psi_{h}^{-1}(1, M)$ has a compactly supported symbol and therefore

$$
\left\|\left[A_{N}, A\right] u\right\|_{L^{2}(M)}=\mathcal{O}\left(h^{1-r-\frac{k}{4}}\right) .
$$

By the choice of $A$ and $A_{N}$ we further have that

$$
\left\|A A_{N} u\right\|_{L^{2}(M)}=\mathcal{O}\left(h^{1-r-\frac{k}{4}}\right) .
$$

Thus, if follows by induction with respect to $N$ that

$$
\left(\prod_{j=1}^{N} A_{j}\right)(A u)=\mathcal{O}_{L^{2}(M)}\left(h^{N-r-\frac{k}{4}}\right), h \rightarrow 0 .
$$


Therefore $A u \in I_{h}^{r}(M, \Lambda)$.

To prove the converse, let $B \in \Psi_{h}^{0}(1, M)$ have a compactly supported symbol and satisfy $\left(x_{0}, \xi_{0}\right) \notin W F_{h}(B A-I)$. Then $\left(x_{0}, \xi_{0}\right) \notin W F_{h}(B A u-u)$. From the first part of the proof, we have that $B A u \in I_{h}^{r}(M, \Lambda)$. Let, now, $P \in \Psi_{h}^{0}(1, M)$ have symbol supported in a sufficiently small neighborhood of $\left(x_{0}, \xi_{0}\right) \in \Lambda$ so that $P B A u-P u=\mathcal{O}\left(h^{\infty}\right)$ in $C^{\infty}(M)$. Since again $P B A u \in I_{h}^{r}(M, \Lambda)$, we have that $\left(\prod_{j=1}^{N} A_{j}\right)(P u)=\mathcal{O}_{L^{2}}\left(h^{N-r-\frac{k}{4}}\right), h \rightarrow 0$, for any set of operators $\left(A_{j}\right)_{j=1}^{N}, N \in \mathbb{N}$, as in (17). Thus $P u \in I_{h}^{r}(M, \Lambda)$ for every $P \in \Psi_{h}^{0}(1, M)$ with symbol supported in a sufficiently small neighborhood of any point $\left(x_{0}, \xi_{0}\right) \in \Lambda$. The compactness of the supports of the operators $\left(A_{j}\right)_{j=1}^{N}, N \in \mathbb{N}$, now allows us to find $P_{j} \in$ $\Psi_{h}^{0}(1, M), j=1, \ldots, J, J \in \mathbb{N}$, such that $P_{j} u \in I_{h}^{r}(M, \Lambda), j=1, \ldots, J$, and $\sum_{j=1}^{J} \sigma\left(P_{j}\right)=1$ on $\cup_{k=1}^{N} \operatorname{supp} \sigma\left(A_{k}\right)$. Using the calculus of semi-classical pseudodifferential operators, we further obtain

$$
\mathcal{O}_{L^{2}(M)}\left(h^{N-r-\frac{k}{4}}\right)=\left(\prod_{k=1}^{N} A_{k}\right)\left(\sum_{j=1}^{J} P_{j}\right) u=\left(\prod_{k=1}^{N} A_{k}\right) u+\mathcal{O}_{L^{2}(M)}\left(h^{\infty}\right),
$$

which completes the proof.

The proof in the case of an operator $A \in \Psi_{h}^{0, k}\left(T^{*} M\right), k \in \mathbb{R}$ is analogous.

We shall now characterize semi-classical Fourier integral distributions microlocally. We have the following

Theorem 1 Let $\Lambda \subset T^{*} \mathbb{R}^{n}$ be a Lagrangian submanifold and let $\gamma \in \Lambda$. Let $\varphi$ be a nondegenerate phase function in an open set $V \subset \mathbb{R}^{n+m}, m \in \mathbb{N}_{0}$, such that $\Lambda=\Lambda_{\varphi}$ in a neighborhood of $\gamma$. If $a \in S_{n+m}^{r+\frac{m}{2}+\frac{n}{4}}(1)$ is such that $\operatorname{supp} a \Subset V$, then $I(a, \varphi) \in I_{h}^{r}\left(\mathbb{R}^{n}, \Lambda\right)$.

Conversely, if $u \in I_{h}^{r}\left(\mathbb{R}^{n}, \Lambda\right)$ microlocally near $\gamma$, then for every non-degenerate phase function $\varphi$ in an open set $V \subset \mathbb{R}^{n+m}, m \in \mathbb{N}_{0}$, such that $\Lambda=\Lambda_{\varphi}$ near $\gamma$, there exists $a \in S_{n+m}^{r+\frac{m}{2}+\frac{n}{4}}(1)$ with $\operatorname{supp} a \Subset V$ such that $u=I(a, \varphi)$ microlocally near $\gamma$.

Proof: Let $\gamma$ have canonical coordinates $\left(x_{0}, \xi_{0}\right)$ and let us first assume that $\Lambda$ is transverse to the section $\xi=\xi_{0}$ at $\gamma$. Then there exists an open neighborhood $U \subset T^{*} \mathbb{R}^{n}$ of $\gamma$ such that $\pi_{\xi}: \Lambda \cap U \ni(x, \xi) \mapsto \xi \in \mathbb{R}^{n}$ in canonical coordinates is a local diffeomorphism. Let $H \in C_{b}^{\infty}\left(\mathbb{R}^{n} ; \mathbb{R}\right)$ be chosen such that, perhaps after adjusting $V, \Lambda_{\varphi}=\left\{\left(H^{\prime}(\xi), \xi\right): \xi \in W\right\}$ for some bounded open set $W \subset \mathbb{R}^{n}$. For $\xi \in \mathbb{R}^{n}$ consider

$$
\widehat{I(a, \varphi)}(\xi)=\iint e^{\frac{i}{h}(\varphi(x, \theta)-\langle x, \xi\rangle)} a(x, \theta) d \theta d x .
$$

For $\xi \notin W$ integration by parts in $(x, \theta)$ gives

$$
\widehat{I(a, \varphi)}=\mathcal{O}\left(h^{\infty}\right) \text { in } C_{c}^{\infty}\left(W^{c}\right) .
$$


Let, now, $\bar{\xi} \in W$. Then the function $\Phi(x, \theta ; \bar{\xi})=\varphi(x, \theta)-\langle x, \bar{\xi}\rangle$ has a critical point at $(\bar{x}(\bar{\xi}), \bar{\theta}(\bar{\xi}))$ which is the inverse image in $C_{\varphi}$ under $j_{\varphi}$ of the point $\left(H^{\prime}(\bar{\xi}), \bar{\xi}\right)$. Using integration by parts again, we obtain that, up to a term which is $\mathcal{O}\left(h^{\infty}\right)$ in $C_{c}^{\infty}(W)$,

$$
\widehat{I(a, \varphi)}(\bar{\xi}) \equiv \iint e^{\frac{i}{h}(\varphi(x, \theta)-\langle x, \bar{\xi}\rangle)} a(x, \theta) \chi(x-\bar{x}(\bar{\xi}), \theta-\bar{\theta}(\bar{\xi})) d \theta d x,
$$

where $\chi \in C_{c}^{\infty}\left(\mathbb{R}^{n+m}\right)$ is equal to 1 on a neighborhood of 0 .

To prove that the critical point is non-degenerate, let $v$ be in the kernel of

$$
\Phi_{x \theta}^{\prime \prime}\left(\bar{x}(\bar{\xi}), \bar{\theta}(\bar{\xi}) ; \xi_{0}\right)=\left[\begin{array}{ll}
\varphi_{x x}^{\prime \prime}(\bar{x}(\bar{\xi}), \bar{\theta}(\bar{\xi})) & \varphi_{x \theta}^{\prime \prime}(\bar{x}(\bar{\xi}), \bar{\theta}(\bar{\xi})) \\
\varphi_{\theta x}^{\prime \prime}(\bar{x}(\bar{\xi}), \bar{\theta}(\bar{\xi})) & \varphi_{\theta \theta}^{\prime \prime}(\bar{x}(\bar{\xi}), \bar{\theta}(\bar{\xi}))
\end{array}\right] .
$$

Then

$$
v \in \operatorname{ker}\left(\varphi_{x \theta}^{\prime \prime}(\bar{x}(\bar{\xi}), \bar{\theta}(\bar{\xi})) \quad \varphi_{\theta \theta}^{\prime \prime}(\bar{x}(\bar{\xi}), \bar{\theta}(\bar{\xi}))\right)
$$

and therefore

$$
v \in T_{(\bar{x}(\bar{\xi}), \bar{\theta}(\bar{\xi}))} C_{\varphi} .
$$

We also have that

$$
v \in \operatorname{ker}\left(\varphi_{x x}^{\prime \prime}(\bar{x}(\bar{\xi}), \bar{\theta}(\bar{\xi})) \quad \varphi_{x \theta}^{\prime \prime}(\bar{x}(\bar{\xi}), \bar{\theta}(\bar{\xi}))\right)
$$

and since $j_{\varphi}$ and $\pi_{\xi}$ are diffeomorphisms, it follows that $v=0$. Hence

$$
\text { the matrix } \Phi_{x \theta}^{\prime \prime}(\bar{x}(\bar{\xi}), \bar{\theta}(\bar{\xi}) ; \bar{\xi}) \text { is non-singular. }
$$

We can therefore apply the method of stationary phase to the integral (91) and obtain

$$
\widehat{I(a, \varphi)}(\bar{\xi}) \sim e^{\frac{i}{h} \Phi(\bar{x}(\bar{\xi}), \bar{\theta}(\bar{\xi}) ; \bar{\xi})} \sum_{k=0}^{\infty} h^{k+\frac{n}{2}+\frac{m}{2}}\left(A_{2 k}\left(D_{x, \theta}\right) a\right)(\bar{x}(\bar{\xi}), \bar{\theta}(\bar{\xi})),
$$

where $A_{2 k}(D)$ are differential operators of orders $\leq 2 k$, respectively.

The Implicit Function Theorem and (10) now imply that, perhaps after shrinking $W$ around $\bar{\xi}, \bar{x}, \bar{\theta} \in C^{\infty}(W)$. We further adjust $W$ so that $\bar{x}, \bar{\theta} \in C_{b}^{\infty}(W)$. Thus

$$
\Phi_{\xi}^{\prime}(\bar{x}(\xi), \bar{\theta}(\xi) ; \xi)=-H^{\prime}(\xi), \xi \in W
$$

and therefore, by adding a constant to $H$ if necessary, we can assume that $\Phi(\bar{x}(\xi), \bar{\theta}(\xi) ; \xi)=$ $-H(\xi)$ for $\xi \in W$. We also have that for every $k, A_{2 k}\left(D_{x, \theta}\right)(a)(\bar{x}(\cdot), \bar{\theta}(\cdot)) \in S_{n}^{r+\frac{m}{2}+\frac{n}{4}}(1)$. Thus, with $A \in S_{n}^{r-\frac{n}{4}}(1), A \sim \sum_{k=0}^{\infty} h^{k+\frac{n}{2}+\frac{m}{2}}\left(A_{2 k}\left(D_{x, \theta}\right) a\right)(\bar{x}(\cdot), \bar{\theta}(\cdot)), A=\mathcal{O}\left(h^{\infty}\right)$ in $S_{n}^{r-\frac{n}{4}}$ (1) outside $W$, we obtain, from (18), (9), and (11), that

$$
\widehat{I(a, \varphi)}(\xi)=e^{-\frac{i H(\xi)}{h}} A(\xi) .
$$

Now, the ideal of smooth functions vanishing on $\Lambda_{\varphi}$ is generated by the symbols $a_{j}(x, \xi)=$ $x_{j}-H_{\xi_{j}}^{\prime}(\xi), j=1, \ldots, n$. Since $I(a, \varphi)$ has compact support, by adjusting $V$ without 
changing $I(a, \varphi)$, we can assume that $\Lambda_{\varphi}$ is compact and we can choose $\chi \in C_{c}^{\infty}\left(T^{*} \mathbb{R}^{n}\right)$ equal to 1 on a neighborhood of $\Lambda_{\varphi}$. Then $\tilde{a}_{j}=\chi a_{j} \in S_{2 n}(1), j=1, \ldots, n$ vanish on $\Lambda_{\varphi}$. By the calculus of pseudodifferential operators, we have that $O p_{h}\left(\tilde{a}_{j}\right) I(a, \varphi)=O p_{h}\left(a_{j}\right) I(a, \varphi)+$ $E_{j} I(a, \varphi)$, where $E_{j} I(a, \varphi)=\mathcal{O}\left(h^{\infty}\right), h \rightarrow 0$ in $C_{c}\left(\mathbb{R}^{n}\right)$.

$$
\begin{gathered}
\mathcal{O}\left(h^{\infty}\right)+\left\|\left(O p_{h}\left(\tilde{a}_{j}\right)\right)^{\alpha}(I(a, \varphi))\right\|_{L^{2}\left(\mathbb{R}^{n}\right)}=\left\|\left(x-H^{\prime}(h D)\right)^{\alpha}(I(a, \varphi))\right\|_{L^{2}\left(\mathbb{R}^{n}\right)} \\
=\frac{1}{(2 \pi h)^{\frac{n}{2}}}\left\|\left(-h D-H^{\prime}\right)^{\alpha} \widehat{I(a, \varphi)}\right\|_{L^{2}\left(\mathbb{R}^{n}\right)}=\mathcal{O}\left(h^{|\alpha|-r-\frac{n}{4}}\right), \alpha \in \mathbb{N}^{n}, h \rightarrow 0 .
\end{gathered}
$$

Thus $I(a, \varphi) \in I_{h}^{r}\left(\mathbb{R}^{n}, \Lambda\right)$.

We remark here that the same argument will allow us in similar situations to use in condition (7) symbols, which do not belong to the class $S(1)$ and below we will do so without repeating this argument.

We now turn to proving the converse. Let $U, H, W$, and $V$ be further chosen so that (4) and (5) hold and $W$ is bounded. Extend $H$ to a function in $C_{b}^{\infty}\left(\mathbb{R}^{n} ; \mathbb{R}\right)$. Let $P \in \Psi_{h}^{0}\left(1, \mathbb{R}^{n}\right)$ satisfy (11) and set $\tilde{u}=P u$. The symbols $a_{j}(x, \xi)=x_{j}-H_{\xi_{j}}^{\prime}(\xi), j=1, \ldots, n$ vanish on $\Lambda_{\varphi} \cap U$, and therefore we obtain from (17) that

$$
\left\|\left(x-H^{\prime}(h D)\right)^{\alpha}(\tilde{u})\right\|_{L^{2}\left(\mathbb{R}^{n}\right)}=\mathcal{O}\left(h^{|\alpha|-r-\frac{n}{4}}\right), \alpha \in \mathbb{N}^{n}, h \rightarrow 0
$$

and hence, after taking the Fourier transform,

$$
\left\|\left(-h D-H^{\prime}\right)^{\alpha} \hat{\tilde{u}}\right\|_{L^{2}\left(\mathbb{R}^{n}\right)}=\mathcal{O}\left(h^{|\alpha|+\frac{n}{4}-r}\right), \alpha \in \mathbb{N}^{n}, h \rightarrow 0
$$

Substituting $\hat{\tilde{u}}(\xi)=e^{-\frac{i H(\xi)}{h}} v(\xi)$ in (12), we obtain

$$
\begin{aligned}
\left\|(h D)^{\alpha} v\right\|_{L^{2}\left(\mathbb{R}^{n}\right)} & =\mathcal{O}\left(h^{|\alpha|+\frac{n}{4}-r}\right), \alpha \in \mathbb{N}^{n}, h \rightarrow 0, \\
\left\|D^{\alpha} v\right\|_{L^{2}\left(\mathbb{R}^{n}\right)} & =\mathcal{O}\left(h^{\frac{n}{4}-r}\right), \alpha \in \mathbb{N}^{n}
\end{aligned}
$$

As in the previous case, this implies that

$$
v \in S_{n}^{r-\frac{n}{4}}(1)
$$

Let $\Phi(x, \theta ; \xi)=\varphi(x, \theta)-\langle x, \xi\rangle, \xi \in W,(x, \theta) \in V$. Choose $\bar{\xi} \in W$, and let $(\bar{x}(\bar{\xi}), \bar{\theta}(\bar{\xi})) \in$ $C_{\varphi}$ be the critical point of $\Phi(\cdot, \cdot \cdot ; \bar{\xi})$. Let $M \Subset V$ be a neighborhood of $(\bar{x}(\bar{\xi}), \bar{\theta}(\bar{\xi}))$ such that sgn $\Phi^{\prime \prime}$ is constant on $M$ and let $\psi \in C^{\infty}\left(M ; \mathbb{R}^{n}\right)$ be such that $\psi(x, \theta)=\varphi_{x}^{\prime}(x, \theta)$ on $C_{\varphi}$. Define

$$
a_{0}(x, \theta)=\frac{1}{(2 \pi h)^{\frac{n}{4}+\frac{m}{2}}}\left(e^{-\frac{i \pi}{4} \operatorname{sgn} \Phi_{x \theta}^{\prime \prime}}\left|\operatorname{det} \Phi_{x \theta}^{\prime \prime}\right|^{\frac{1}{2}} v\right) \circ \psi(x, \theta) \text { for }(x, \theta) \in M .
$$

Then, by the first part of the proof, we have that

$$
e^{-\frac{i H}{h}} v-I \widehat{\left(a_{0}, \varphi\right)}=\mathcal{O}(h), h \rightarrow 0, \text { in } S_{n}^{r-\frac{n}{4}}(1) .
$$


Iterating this process, we obtain a sequence of symbols $a_{l} \in S_{n}^{r+\frac{m}{2}+\frac{n}{4}}(1)$ such that, if we denote $U_{s}=I\left(\sum_{l=0}^{s} h^{l} a_{l}, \varphi\right), s \in \mathbb{N}_{0}$ we have that

$$
e^{-\frac{i H}{h}} v-\hat{U}_{s}=\mathcal{O}\left(h^{s+1}\right), h \rightarrow 0, \text { in } S_{n}^{r-\frac{n}{4}}(1) .
$$

Therefore, if we choose an asymptotic sum $a \in S_{n+m}^{r+\frac{m}{2}+\frac{n}{4}}(1)$ of $\sum_{k=0}^{\infty} h^{k} a_{k}$, we obtain $\tilde{u}=$ $I(a, \varphi)$ microlocally near $\gamma$.

Let, now, $\Lambda \subset T^{*} \mathbb{R}^{n}$ be any Lagrangian submanifold and assume that the coordinates have been chosen in such a way that $\mu=T_{\gamma}^{*} \Lambda$ has the form (6). Choose a real symmetric matrix $A_{\Lambda}=\left[\begin{array}{cc}0_{k \times k} & 0 \\ 0 & D_{\Lambda(n-k) \times(n-k)}\end{array}\right]$ such that

$$
\operatorname{det}\left(B+D_{\Lambda}\right) \neq 0
$$

Let $\tilde{\Lambda}=\left\{\left(x, \xi+A_{\Lambda} x\right):(x, \xi) \in \Lambda\right\}$ and let $\tilde{\gamma}=\left(x_{0}, \xi_{0}+A_{\Lambda} x_{0}\right)=\left(x_{0}, \eta_{0}\right)$, where $\left(x_{0}, \xi_{0}\right)$ are the coordinates of $\gamma$. Then, if $\varphi \in C^{\infty}(V ; \mathbb{R}), V \subset \mathbb{R}^{n+m}, m \in \mathbb{N}_{0}$, is a non-degenerate phase function which parameterizes $\Lambda$ near $\gamma$, it is clear that $\tilde{\varphi}(x, \theta)=\frac{1}{2}\left\langle A_{\Lambda} x, x\right\rangle+\varphi(x, \theta)$ is a non-degenerate phase function which parameterizes $\tilde{\Lambda}$ near $\tilde{\gamma}$.

Let $\tilde{\mu}=T_{\tilde{\gamma}} \tilde{\Lambda}$. It is easy to see that $\tilde{\mu}=\left\{\left(0, x^{\prime \prime} ; \xi^{\prime},\left(B+D_{\Lambda}\right) x^{\prime \prime}\right)\right\}$ and it then follows from (14) that $\tilde{\Lambda}$ is transverse to the constant section $\eta=\eta_{0}$ at $\tilde{\gamma}$.

Let $u \in I_{h}^{r}\left(\mathbb{R}^{n}, \Lambda\right)$ microlocally near $\gamma$ and let $A_{k} \in \Psi_{h}^{0}(1, X), k=1, \ldots, N$, be such that $\left.\sigma_{0}\left(A_{k}\right)\right|_{\Lambda}=0$. From (17) we have that

$$
\left(\prod_{k=1}^{N} e^{\frac{i}{h}\left\langle A_{\Lambda} \cdot, \cdot\right\rangle} A_{k} e^{-\frac{i}{h}\left\langle A_{\Lambda} \cdot \cdot, \cdot \cdot\right\rangle}\right) e^{\frac{i}{h}\left\langle A_{\Lambda} \cdot \cdot, \cdot \cdot\right\rangle} u=\mathcal{O}_{L^{2}\left(\mathbb{R}^{n}\right)}\left(h^{N-r}\right), h \rightarrow 0 .
$$

Let $K(z, t)=\frac{1}{(2 \pi h)^{n}} e^{-\frac{i}{h}\left\langle A_{\Lambda} z, z\right\rangle} \int e^{\frac{i}{h}\langle z-t, \xi\rangle} a(z, \xi) d \xi e^{\frac{i}{h}\left\langle A_{\Lambda} t, t\right\rangle}$ and consider

$$
\begin{aligned}
b(w, \tau) & =\int e^{\frac{i}{h} y \cdot \tau} K(w, w+y) d y \\
& =\frac{1}{(2 \pi h)^{n}} \iint e^{\frac{i}{h}\left(\langle y, \tau-\xi\rangle-\left\langle A_{\Lambda} w, w\right\rangle+\left\langle A_{\Lambda}(w+y),(w+y)\right\rangle\right)} a(w, \xi) d \xi d y .
\end{aligned}
$$

We apply the method stationary phase to the above integral. The phase has a critical point at $\left(y_{0}, \xi_{0}\right)=\left(0, \tau+A_{\Lambda} w\right)$ and the Hessian of the phase at the critical point is $\left[\begin{array}{cc}A_{\Lambda} & -I \\ -I & 0\end{array}\right]$, which has determinant 1 and signature 0 . Therefore $b \sim \sum_{k=0}^{\infty} h^{k} b_{k}$ with $b_{k} \in S_{2 n}(1)$ and

$$
b_{0}(w, \tau)=a\left(w, \tau+A_{\Lambda} w\right) .
$$

This implies that $b \in S_{2 n}(1)$ and $B_{k}=e^{\frac{i}{h}\left\langle A_{\Lambda} \cdot \cdot \cdot\right\rangle} A_{k} e^{-\frac{i}{h}\left\langle A_{\Lambda} \cdot \cdot, \cdot \cdot\right\rangle} \in \Psi_{h}^{0}(1, X)$. From (15) we then have that $\left.\sigma_{0}\left(B_{k}\right)\right|_{\tilde{\Lambda}}=0, k=1, \ldots, N$. We can now apply the first part of the proof of this theorem and we have that $e^{\frac{i}{h}\left\langle A_{\Lambda} \cdot \cdot\right\rangle} u=I(a, \tilde{\varphi}), a \in S_{n+m}^{r+\frac{m}{2}+\frac{n}{4}}(1)$. Therefore $u=I(a, \varphi)$. 
The converse follows from reversing this argument.

Remark. Let $u \in I_{h}^{r}(M, \Lambda)$. Then Theorem 1 and Lemma 1 implies that for any $P \in$ $\Psi_{h}^{0}(1, M)$ with a compactly supported symbol $P u$ is given by a finite sum of oscillatory integrals of the form $h^{-r} I(a, \varphi)$, where $a \in S(1)$ and $\varphi$ is a non-degenerate phase function such that $\Lambda=\Lambda_{\varphi}$ near a point in $\Lambda$.

Following this remark, we see that after taking a locally finite partition of unity $\left(a_{j}\right)_{j=1}^{\infty} \subset$ $C_{c}^{\infty}\left(T^{*} M\right)$ such that $\sum_{j=1}^{\infty} a_{j}=1$ on $\Lambda$ and applying an integration by parts argument, as in [3], Chapter 7, we have

Lemma 6 If $u \in I_{h}^{r}(M, \Lambda)$, then $W F_{h}^{f}(u) \subset \Lambda$.

\subsection{Generalization of Egorov's Theorem}

We now prove the following generalization of Egorov's Theorem to manifolds of unequal dimensions:

Lemma 7 Let $X_{j}, j=1,2$, be smooth manifolds. Let $\sigma_{j}$ be the canonical symplectic form on $T^{*} X_{j}$, and $\pi_{j}: T^{*} X_{1} \times T^{*} X_{2} \rightarrow T^{*} X_{j}$ the projection onto the $j$ - th factor. Let

$$
\Lambda \subset T^{*} X_{1} \times T^{*} X_{2}
$$

be a Lagrangian submanifold of

$$
\left(T^{*} X_{1} \times T^{*} X_{2}, \pi_{1}^{*} \sigma_{1}+\pi_{2}^{*} \sigma_{2}\right)
$$

such that $\left.\pi_{2}\right|_{\Lambda}$ is an immersion. Let $F \in \mathcal{I}_{h}^{r}\left(X_{1} \times X_{2}, \Lambda\right), r \in \mathbb{R}$, have a non-vanishing principal symbol at $\left(\rho_{1}, \rho_{2}\right) \in \Lambda$.

Then for every $A \in \Psi_{h}^{0}\left(1, X_{1}\right)$ with symbol supported in a sufficiently small neighborhood of $\rho_{1}$ there exists $B \in \Psi_{h}^{0}\left(1, X_{2}\right)$ with symbol supported in a sufficiently small neighborhood of $\rho_{2}$ such that

$$
A F \equiv F B \text { near }\left(\rho_{1}, \rho_{2}\right)
$$

and

$$
i^{n_{2}}\left(\left.\pi_{2}\right|_{\Lambda}\right)^{*} \sigma_{0}(B)=i^{n_{1}}\left(\left.\pi_{1}\right|_{\Lambda}\right)^{*} \sigma_{0}(A) .
$$

Remark: Strictly speaking we have not defined a symbol of a Fourier integral operator given in Definition 3. However, the proof of Theorem 1 shows that the non-vanishing of the amplitude given there is invariantly defined.

Proof: By a partition of unity we can reduce the proof to the local case where $X_{j} \subset \mathbb{R}^{n_{j}}$, $T^{*} X_{j}$ is trivial for $j=1,2, F=\int e^{\frac{i}{h} \varphi(x, z, \theta)} u(x, z, \theta) d \theta$, where $\varphi$ is a non-degenerate phase function in a neighborhood of $\left(x_{0}, z_{0}, \theta_{0}\right) \in X_{1} \times X_{2} \times \mathbb{R}^{m}$ for some $m \in \mathbb{N}_{0}$ such that $\Lambda \cap U=\Lambda_{\varphi}$ for an open set $U$ with $\left(x_{0}, \xi_{0} ; z_{0}, \eta_{0}\right) \in \Lambda \cap U, u \in S_{n_{1}+n_{2}+m}^{\frac{n_{1}+n_{2}}{4}+\frac{m}{2}+r}(1) \cap C_{c}^{\infty}\left(\mathbb{R}^{n_{1}+n_{2}+m}\right)$, 
$u \sim \sum_{k=0}^{\infty} h^{k+\frac{n_{1}+n_{2}}{4}+\frac{m}{2}+r} u_{k}, u_{k} \in S_{n_{1}+n_{2}+m}^{0}(1)$, and $A=\int_{\mathbb{R}^{n_{1}}} e^{\frac{i}{h}\langle x-y, \xi\rangle} a(x, \xi) d \xi$ with $a \in$ $S_{2 n_{1}}^{0}(1) \cap C_{c}^{\infty}\left(\mathbb{R}^{2 n_{1}}\right), a \sim \sum_{k=0}^{\infty} h^{k} a_{k}, a_{k} \in S_{2 n_{1}}^{0}(1)$.

Let $\Phi(y, \xi ; x, z, \theta)=\langle x-y, \xi\rangle+\varphi(y, z, \theta)$. Then $\Phi$ has a critical point

$$
p_{0}(x, z, \theta)=\left(y_{0}(x, z, \theta), \xi_{0}(x, z, \theta)\right)=\left(x, \varphi_{x}^{\prime}(x, z, \theta)\right) .
$$

The Hessian of $\Phi$ is

$$
\Phi^{\prime \prime}\left(y_{0}(x, z, \theta), \xi_{0}(x, z, \theta) ; x, z, \theta\right)=\left[\begin{array}{cc}
\varphi_{x x}^{\prime \prime}(x, z, \theta) & -I \\
-I & 0
\end{array}\right]
$$

and has determinant 1 and signature 0 .

Let $\Psi(w, \eta ; x, z, \theta)=\varphi(x, w, \theta)+\langle w-z, \eta\rangle$. Then $\Psi$ has a critical point

$$
q_{0}(x, z, \theta)=\left(w_{0}(x, z, \theta), \eta_{0}(x, z, \theta)\right)=\left(z,-\varphi_{z}^{\prime}(x, z, \theta)\right) .
$$

The Hessian of $\Psi$ is

$$
\Psi^{\prime \prime}\left(w_{0}(x, z, \theta), \eta_{0}(x, z, \theta) ; x, z, \theta\right)=\left[\begin{array}{cc}
\varphi_{z z}^{\prime \prime}(x, z, \theta) & I \\
I & 0
\end{array}\right]
$$

and has determinant 1 and signature 0 .

We define

$$
\begin{aligned}
g_{p_{0}(x, z, \theta)}(p)= & \Phi(p ; x, z, \theta)-\Phi\left(p_{o}(x, z, \theta) ; x, z, \theta\right) \\
& -\frac{\left\langle\Phi^{\prime \prime}\left(p_{0}(x, z, \theta) ; x, z, \theta\right)\left(p-p_{0}(x, z, \theta)\right), p-p_{0}(x, z, \theta)\right\rangle}{2}
\end{aligned}
$$

and

$$
\begin{aligned}
f_{q_{0}(x, z, \theta)}(q)= & \Psi(q ; x, z, \theta)-\Psi\left(q_{o}(x, z, \theta) ; x, z, \theta\right) \\
& -\frac{\left\langle\Psi^{\prime \prime}\left(q_{0}(x, z, \theta) ; x, z, \theta\right)\left(q-q_{0}(x, z, \theta)\right), q-q_{0}(x, z, \theta)\right\rangle}{2} .
\end{aligned}
$$

For $j \in \mathbb{N}_{0}$ and $c \in C_{c}^{\infty}\left(\mathbb{R}^{2 m}\right)$, set

$$
\begin{aligned}
\left(L_{j}^{l}(c u)\right)(x, z, \theta)=\sum_{\nu-\mu=j} \sum_{2 \nu \geq 3 \mu} \frac{\left\langle\left(\Phi^{\prime \prime}\left(p_{0}(x, z, \theta) ; x, z, \theta\right)\right)^{-1} D, D\right\rangle^{\nu}}{i^{j} 2^{\nu} \mu ! \nu !} \\
\left(g_{p_{0}(x, z, \theta)}^{\mu} c(x, \cdot \cdot) u(\cdot, z, \theta)\right)\left(p_{0}(x, z, \theta)\right) .
\end{aligned}
$$

and

$$
\begin{aligned}
\left(L_{j}^{r}(u c)\right)(x, z, \theta)=\sum_{\nu-\mu=j} \sum_{2 \nu \geq 3 \mu} \frac{\left\langle\left(\Psi^{\prime \prime}\left(q_{0}(x, z, \theta) ; x, z, \theta\right)\right)^{-1} D, D\right\rangle^{\nu}}{i^{j} 2^{\nu} \mu ! \nu !} \\
\left(f_{q_{0}(x, z, \theta)}^{\mu} c(\cdot, \cdot \cdot) u(x, \cdot, \theta)\right)\left(q_{0}(x, z, \theta)\right) .
\end{aligned}
$$


Now, since $\left.\pi_{2}\right|_{\Lambda}$ is an immersion, it follows from the Inverse Function Theorem, that there exists $\kappa \in C^{\infty}\left(T^{*} \mathbb{R}^{n_{2}} ; T^{*} \mathbb{R}^{n_{1}} \times T^{*} \mathbb{R}^{n_{2}}\right)$, such that $\left.\kappa \circ \pi_{2}\right|_{\Lambda}=\left.\mathrm{id}\right|_{\Lambda}$. Let, now, $b_{0} \in C_{c}^{\infty}\left(\mathbb{R}^{2 n_{2}}\right)$ be supported near $\rho_{2}$ and satisfy

$$
b_{0}=i^{n_{1}-n_{2}} \kappa^{*}\left(\left.\pi_{1}\right|_{\Lambda}\right)^{*} a_{0} .
$$

Then $i^{n_{2}} j_{\varphi}^{*}\left(\left.\pi_{2}\right|_{\Lambda}\right)^{*} b_{0}-i^{n_{1}} j_{\varphi}^{*}\left(\left.\pi_{1}\right|_{\Lambda}\right)^{*} a_{0}$ vanishes on $C_{\varphi}$ and since $\varphi$ is a non-degenerate phase function, it follows that there exist $c_{j}^{0} \in C_{c}^{\infty}\left(\mathbb{R}^{n_{1}+n_{2}+m}\right), j=1, \ldots, m$, such that

$$
i^{n_{1}} j_{\varphi}^{*}\left(\left.\pi_{1}\right|_{\Lambda}\right)^{*} a_{0}-i^{n_{2}} j_{\varphi}^{*}\left(\left.\pi_{2}\right|_{\Lambda}\right)^{*} b_{0}=\sum_{j=1}^{m} \varphi_{\theta_{j}}^{\prime} c_{j}^{0} .
$$

For every $k>0$ we now choose $b_{k} \in C_{c}^{\infty}\left(\mathbb{R}^{2 n_{2}}\right)$ in such a way that

$$
\left(i^{n_{1}} \sum_{\alpha+\beta+\gamma=k} L_{\alpha}^{l}\left(a_{\gamma} u_{\beta}\right)-i^{n_{2}} \sum_{\alpha+\beta+\gamma=k,} L_{\alpha}^{r}\left(u_{\beta} b_{\gamma}\right)-\sum_{l=1}^{m} D_{\theta_{l}}\left(c_{l}^{k-1} u_{0}\right)\right)_{\left.\right|_{C_{\varphi}}}=0
$$

and choose $c_{l}^{k} \in C_{c}^{\infty}\left(\mathbb{R}^{n_{1}+n_{2}+m}\right), j=1, \ldots, m$, such that

$$
i^{n_{1}} \sum_{\alpha+\beta+\gamma=k} L_{\alpha}^{l}\left(a_{\gamma} u_{\beta}\right)-i^{n_{2}} \sum_{\alpha+\beta+\gamma=k,} L_{\alpha}^{r}\left(u_{\beta} b_{\gamma}\right)-\sum_{l=1}^{m} D_{\theta_{l}}\left(c_{l}^{k-1} u_{0}\right)=\sum_{l=1}^{m} \varphi_{\theta_{l}}^{\prime} c_{l}^{k} u_{0} .
$$

Lastly, let

$$
b \sim \sum_{j=0}^{\infty} h^{j} b_{j}
$$

In the integrals

$$
L(x, z)=A F(x, z)=\frac{1}{(2 \pi h)^{n_{1}}} \iint e^{\frac{i}{h}\langle x-y, \xi\rangle} a(x, \xi) e^{\frac{i}{h} \varphi(y, z, \theta)} u(y, z, \theta) d \theta d y d \xi
$$

and

$$
R(x, z)=F B(x, z)=\frac{1}{(2 \pi h)^{n_{2}}} \iint e^{\frac{i}{h} \varphi(x, w, \theta)} u(x, w, \theta) e^{\frac{i}{h}\langle w-z, \eta\rangle} b(w, \eta) d \theta d w d \eta .
$$

we now apply the method of stationary phase, Theorem 7.7.5 in [4], in the $(y, \xi)$ and the $(w, \eta)$ variables, respectively, and obtain

$$
\begin{aligned}
& L(x, z) \sim i^{n_{1}} \sum_{t=0}^{\infty} h^{t-\frac{n_{1}+n_{2}}{4}-\frac{m}{2}-r} \sum_{j=0}^{t} \sum_{v=0}^{t-j} \int e^{\frac{i}{h} \varphi(x, z, \theta)}\left(L_{j}^{l}\left(a_{t-j-v} u_{v}\right)\right)(x, z, \theta) d \theta \\
& R(x, z) \sim i^{n_{2}} \sum_{t=0}^{\infty} h^{t-\frac{n_{1}+n_{2}}{4}-\frac{m}{2}-r} \sum_{j=0}^{t} \sum_{v=0}^{t-j} \int e^{\frac{i}{h} \varphi(x, z, \theta)}\left(L_{j}^{r}\left(u_{v} b_{t-j-v}\right)\right)(x, z, \theta) d \theta .
\end{aligned}
$$


By the choice of $b_{0}$ we have

$$
\begin{aligned}
& \int e^{\frac{i}{h} \varphi(x, z, \theta)} u_{0}(x, z, \theta)\left[i^{n_{1}} a_{0}\left(x, \varphi_{x}^{\prime}(x, z, \theta)\right)-i^{n_{2}} b_{0}\left(z,-\varphi_{z}^{\prime}(x, z, \theta)\right)\right] d \theta \\
& =\int e^{\frac{i}{h} \varphi(x, z, \theta)} \sum_{j=1}^{m} \varphi_{\theta_{j}}^{\prime}(x, z, \theta) c_{j}^{0}(x, z, \theta) u_{0}(x, z, \theta) d \theta \\
& =-h \int e^{\frac{i}{h} \varphi(x, z, \theta)} \sum_{j=1}^{m} D_{\theta_{j}}\left(c_{j}^{0}(x, z, \cdot) u_{0}(x, z, \cdot)\right)(\theta) d \theta
\end{aligned}
$$

using integration by parts. By the choice of the symbol $b_{1}$ we then have that

$$
A F-F B=\mathcal{O}_{C_{c}^{\infty}\left(\mathbb{R}^{n_{1}+n_{2}}\right)}\left(h^{2-\frac{n_{1}+n_{2}}{4}-\frac{m}{2}-r}\right) .
$$

Iterating this argument, we obtain from the choice of the symbols $b_{k}^{\prime} \mathrm{s}$ that

$$
A F \equiv F B \text { near }\left(\rho_{1}, \rho_{2}\right) \text {. }
$$

Acknowledgements. I would like to thank Victor Ivrii and Maciej Zworski for helpful discussions during the preparation of this article. I would also like to thank Vesselin Petkov for introducing me to the work of his student Laurent Michel, which has helped me complete my work on this article.

\section{References}

[1] C. Gerard, Asymptotique des pôles de la matrice de scattering pour deux obstacles strictement convexes, Mémoires de la Société Mathématique de France 116 (31) (1988).

[2] M. Dimassi and J. Sjöstrand, Spectral Asymptotics in the Semi-Classical Limit, Cambridge University Press, Cambridge, 1999.

[3] A. Grigis and J. Sjöstrand, Microlocal Analysis for Differential Operators, Cambridge University Press, Cambridge, 1994.

[4] L. Hörmander, The Analysis of Linear Partial Differential Operators, Springer Verlag, Berlin, 1980.

[5] A. Martinez, An Introduction to Semiclassical and Microlocal Analysis, Springer-Verlag, New York, 2002.

[6] L. Michel, Semi-classical Behavior of the Scattering Amplitude for Trapping Perturbations at Fixed Energy, Ph. D. Dissertation, Université de Bordeaux, 2001.

[7] J. Sjöstrand and M. Zworski, Quantum Monodromy and Semi-classical Trace Formulae, Journal de Mathématiques Pures at Appliquées 81 (1)(2002), 1-33. 